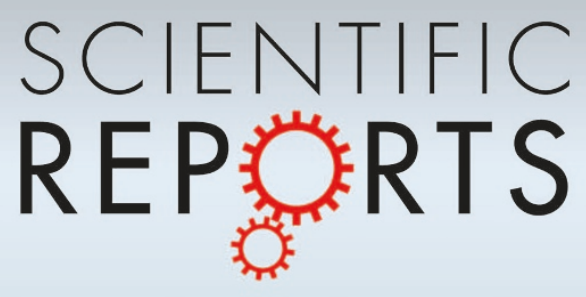

\title{
OPEN A journey from order to disorder - Atom by atom transformation from graphene to a 2D carbon glass
}

SUBJECT AREAS:

STRUCTURAL PROPERTIES

STRUCTURE OF SOLIDS AND

LIQUIDS

\author{
Franz R. Eder' , Jani Kotakoski ${ }^{1,2}$, Ute Kaiser ${ }^{3}$ \& Jannik C. Meyer ${ }^{1,3}$
}

Received

5 December 2013

Accepted

23 January 2014

Published

11 February 2014

\begin{abstract}
${ }^{1}$ Faculty of Physics, University of Vienna, Boltzmanngasse 5, 1090 Vienna, Austria, ${ }^{2}$ Department of Physics, P.O. Box 43, 00014 University of Helsinki, Finland and, ${ }^{3}$ Central Facility for Electron Microscopy, Group of Electron Microscopy of Materials Science, University of Ulm, Albert Einstein Allee 11,89081 Ulm, Germany.
\end{abstract}

Correspondence and requests for materials should be addressed to J.C.M. (jannik.meyer@ univie.ac.at)

One of the most interesting questions in solid state theory is the structure of glass, which has eluded researchers since the early 1900 's. Since then, two competing models, the random network theory and the crystallite theory, have both gathered experimental support. Here, we present a direct, atomic-level structural analysis during a crystal-to-glass transformation, including all intermediate stages. We introduce disorder on a 2D crystal, graphene, gradually, utilizing the electron beam of a transmission electron microscope, which allows us to capture the atomic structure at each step. The change from a crystal to a glass happens suddenly, and at a surprisingly early stage. Right after the transition, the disorder manifests as a vitreous network separating individual crystallites, similar to the modern version of the crystallite theory. However, upon increasing disorder, the vitreous areas grow on the expense of the crystallites and the structure turns into a random network. Thereby, our results show that, at least in the case of a 2D structure, both of the models can be correct, and can even describe the same material at different degrees of disorder.

ince over 80 years, the popular concept of the atomic structure of a glass ${ }^{1}$ has been strongly influenced ${ }^{2,3}$ by the beautiful illustrations of a random network by Zachariasen ${ }^{4}$. However, this theory can not easily be verified, since imaging of the atomic structure of a conventional glass has remained impossible. The direct study of atomic coordinates in a glass has therefore for the most part remained in the realm of theoretical models and computer simulations $s^{5,6}$. Although recent images of the $2 \mathrm{D}$ silica glass ${ }^{7,8}$ revealed vitreous regions, which look remarkably similar to Zachariasen's illustration, also crystalline areas were discovered. Due to small samples and limited statistics, it was impossible to determine whether these crystallites are an intrinsic part of the glass structure or a separate phase depending on the exact growth conditions of the material. Nevertheless, their existence gives much needed credibility for the crystallite theory ${ }^{2}$, which posits that a glass is a disordered arrangement of small crystallite particles separated by a disordered network. In a recent study ${ }^{9}$, also giving support for the crystallite model, the ratio of crystallites was found to be up to $50 \%$ in amorphous sputtered silicon. To finally resolve this issue, one would need a way to directly monitor the glass formation at the atomic level. However, the traditional way to form a glass, i.e., to quench a liquid ${ }^{10,11}$, renders this impossible.

In this study, in contrast to melting and quenching a solid, we gradually introduce disorder into graphene using an electron beam ${ }^{12}$, so that the material remains in the solid state. Although our way to introduce disorder in the crystal differs fundamentally from the traditional method for creating a glass by quenching a melt, we believe that it can lead to new insights into the structures of disordered materials. The two-dimensionality of graphene allows us to directly observe all atoms, and to sidestep the imaging problem of conventional materials. The energy of the electrons is chosen slightly above the damage threshold to keep the rate of transformation slow enough to allow accurate monitoring of every change in structure. The atomic network is altered both via removal of atoms ${ }^{13}$ as well as bond rotations ${ }^{14}$, which occur at random locations and are sufficiently temporarily spaced to be stochastic and mutually independent. The introduced disorder manifests in the formation of non-hexagonal carbon rings. As has been described earlier ${ }^{15,16}$, the graphene lattice can remain flat even upon introduction of pentagons and heptagons. At initial stages of the experiment, small isolated defects appear within the lattice (Fig. 1 a), as has been discussed previously ${ }^{12}$. Here, we focus on the amorphous networks that form under increased irradiation dose, and have not been analyzed so far. Under continuous irradiation, defects grow until they form a vitreous network separating the original crystal into nanometer-sized crystallites (Fig. 1 b), as will be shown in more detail below. During the experiments, also holes appear in the graphene sheet. However, they are created by chemical effects 

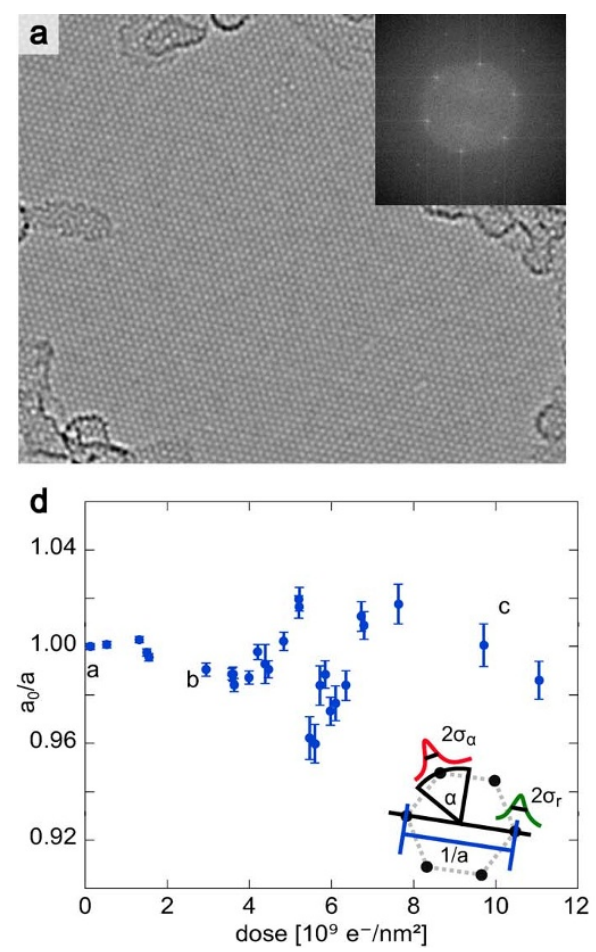
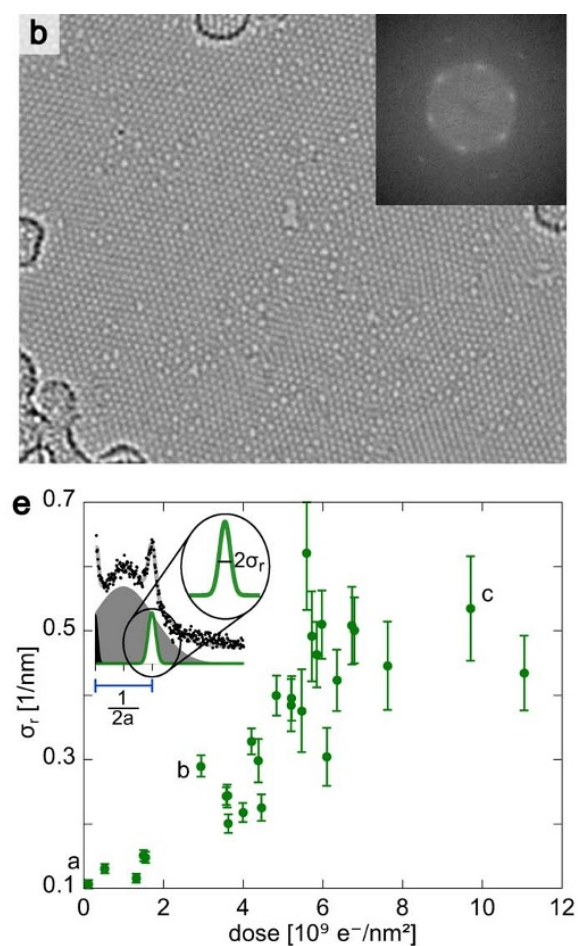

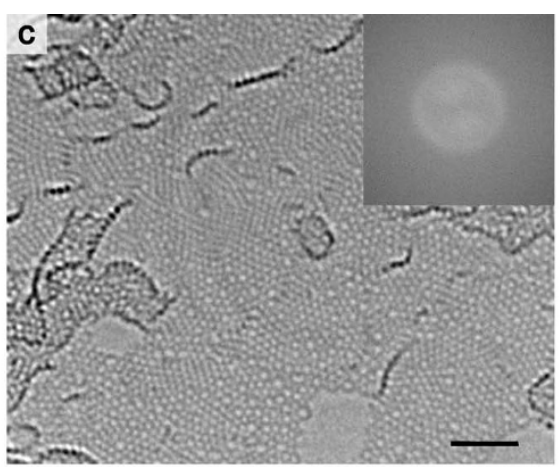

density deficit [\%]

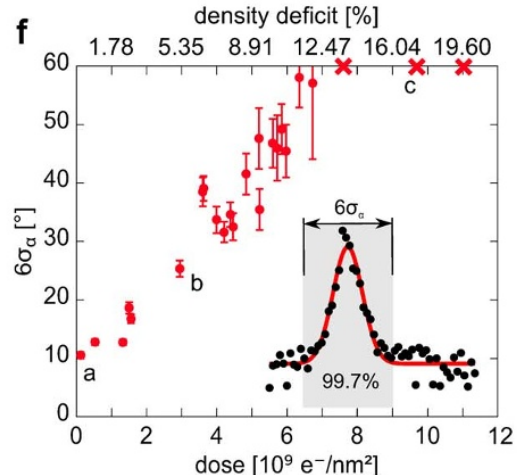

Figure $1 \mid$ Amorphization of graphene and its manifestation in the reciprocal space. Panels $(\mathrm{a}-\mathrm{c})$ show three example TEM images and their corresponding Fourier transformations (FTs, insets in the top right corner) at (a) a low irradiation dose $\left(1.25 \times 10^{8} e^{-} / \mathrm{nm}^{2}\right)$, (b) an intermediate dose $\left(2.94 \times 10^{9} e^{-} / \mathrm{nm}^{2}\right)$ and $(\mathrm{c})$ a high dose $\left(9.36 \times 10^{9} e^{-} / \mathrm{nm}^{2}\right)$. Panels $(\mathrm{d}-\mathrm{f})$ depict the analysis of the FTs: $(\mathrm{d})$ change in the distance between FT peaks in the radial direction $\left(a_{0} / a\right)$, (e) spread of the diffraction peaks in the radial direction $\left(\sigma_{r}\right)$ and (f) the spread of the peak along the ring $\left(6 \sigma_{\alpha}\right)$ as a function of the irradiation dose. The inset in panel (d) shows a schematic presentation of the analysis carried out for panels ( $d-f)$. The insets in panels (e), ( $f$ ) indicate how the FT spectrum was fitted to Gaussians to obtain the presented data. For the last three points in panel (f) (red crosses), the peaks have spread into continuous rings. The second $x$-axis in panel (f) shows the calculated density deficit.

driven by the electron beam ${ }^{13}$, and therefore not considered a part of the disordered structure. At the final stage, the crystallites vanish into the growing network (Fig. $1 \mathrm{c}$ ). Importantly, the structure remains two-dimensional throughout the transformation, providing direct views of the atomic structure at each stage.

In order to quantify the material-wide structural changes, we first apply Fourier analysis on selected atomically resolved TEM images during the transition. At low doses, the sixfold pattern of graphene is clearly visible (see the inset in Fig. 1 a). During the experiment, the peak-to-peak distance remains constant (Fig. $1 \mathrm{~d}$; we attribute the variations to changes in instrumental parameters during the experiment), whereas the peak widths change significantly. The spread in radial direction, shown in Fig. 1 e, displays three different regimes: (1) at low doses, where the material contains isolated defects, it increases only slightly (from point a almost until b), whereas after merging of the defects into the vitreous network, (2) the spread accelerates (between points $b$ and c), (3) finally saturating at the highest doses. The spreading of the peaks corresponds to decreasing size of ordered structures within the sample, and is direct evidence of decreasing crystallite size ${ }^{17}$. Evidently, at the highest doses, the minimum size has already been reached. A more direct measure for the disorder in the structure can be obtained by analyzing the spread of the peaks in azimuthal direction. As can be seen in Fig. $1 \mathrm{f}$, it appears to depend almost linearly on the dose, up to the fully disordered case (peak spread of $60^{\circ}$ ). Comparison between Figs. $1 \mathrm{e}$ and $\mathrm{f}$ shows that the completion of the disorder coincides with the saturation of the decreasing crystallite size. Because the atomic changes imposed by electron irradiation on a sample depend on the acceleration voltage of the TEM, we introduce density deficit as a transferable proxy for the disorder (second $x$-axis in Fig. $1 \mathrm{f}$ ). It can be directly determined experimentally, as described in Ref. 13.
In modern aberration corrected TEM instruments, and for very thin samples, the obtained images can be directly interpreted as the projected atomic structure. However, the exact atomic configurations have been traditionally obtained manually from the images, which is both laborious and error-prone. Therefore, even though amorphization of graphene under a TEM has been reported earlier ${ }^{12}$, the analysis was limited to relatively small isolated defects. To circumvent this problem, we generated an automatic method for extracting the atomic coordinates directly from TEM images. First, we created model TEM images corresponding to carbon rings with different number of atoms (from pentagons to octagons), and then calculated a cross correlation map for each of them for each of the actual TEM images. The maxima within these maps mark the centers of carbon rings within the atomic network, from which the vertices of the polygons (i.e., atomic coordinates) were directly obtained assuming each atom has three neighbors. After obtaining the structures, we optimized them with conjugate gradient energy minimization method (to ensure optimized bond lengths, and to allow slight outof-plane corrugation $\left.s^{18}\right)$. We estimate that inaccuracies in the obtained structures are less than 5\% for non-hexagons, and much lower for the crystalline areas. Moreover, any deviations from the actual structure are random since all user-bias is eliminated, further enhancing the statistics. As shown in the supplementary information, the obtained structures very well match the experimental images. Atomic coordinates obtained with this method provide the basis for our analysis of intermediate stages during the structural transformation.

In Fig. 2, we present examples of atomic structures derived from the TEM images (the supplementary information contains all structures that were used in the present study), and highlight the three regimes that we identified above (crystalline with isolated defects, crystallite glass and random network). Fig. 2 a shows the first regime 


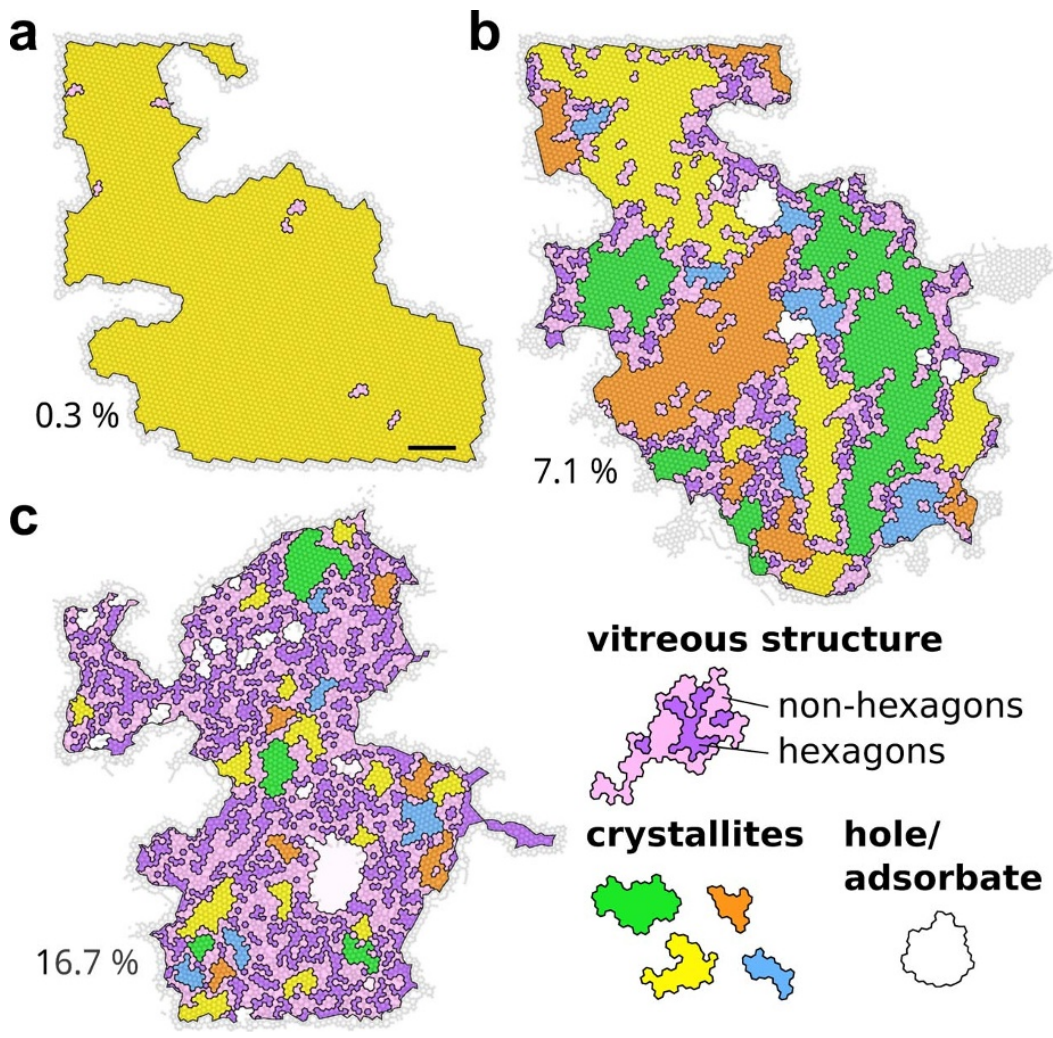

Figure $2 \mid$ Atomic structures derived from TEM images. (a) Initial part of the amorphization (density deficit of $0.3 \%$ ), showing a crystalline lattice with few isolated defects. (b) Nano-crystalline structure showing well isolated crystallites, separated by a vitreous network (deficit of 7.1\%). (c) Random network structure with only a minority of crystallites at the end of the experiment (deficit of 16.7\%). Pink areas denote non-hexagonal rings, violet denotes hexagonal rings that are surrounded by non-hexagonal ones, whereas white areas mark non-resolved structure (typically due to holes or adsorbates). All other colors denote separate crystallites. Crystallites are identified as any hexagonal area that contains at least an area of $3 \times 3$ hexagons, and are considered as separate, if a connection is less than 3 unit cells wide. The scale bar is $2 \mathrm{~nm}$. The supplementary information contains further details on how these maps were generated.

with a few isolated defects. Upon increased irradiation dose, the defects grow and begin to merge. Remarkably, this happens in such a way that isolated crystallites, separated by a vitreous network, are formed. Fig. 2 b shows an example case, where isolated crystallites can be clearly identified. We point out that although this structure somewhat resembles polycrystalline graphene with nanometerrange grains ${ }^{19,20}$, the vitreous network is much wider than typical graphene grain boundaries ${ }^{21-23}$ and covers a significantly higher fraction of the area. Upon further irradiation, the vitreous areas grow at the expense of the crystalline ones, resulting in a structure that has almost no extended hexagonal areas, and is well described by the random network theory. This final state is shown in Fig. 2 c, where the vitreous areas clearly dominate the structure.

In contrast to vitreous oxides like silica, which are built from multi-atomic tetrahedral structural units, the building block in our case is the carbon atom. Therefore, the shortest possible range of disorder in this material is at the interconnection and orientation between adjacent structural units ${ }^{2}$. To quantify this disorder, we compute the distribution of the inter-atomic distances from the atomic coordinates (radial distribution function, $g(r)$ ). This analysis was limited to the intermediate structures to ensure that the statistics for the longer inter-atomic distances ( $r \geq 0.5 \mathrm{~nm}$ ) remain meaningful (the number of holes and other complications in the structure increases during the experiment). Example cases are shown in Fig. 3 $a-c$ for different regimes during the transformation. Although deviation from ideal inter-atomic distances (Fig. 3 a) are already clear close to the crystalline-to-glass transition (Fig. 3 b, see Fig. 3 e for the structure), the disappearance of the long range order only becomes complete after the crystallite structure has been formed (Fig. $3 \mathrm{c}$, see
Fig. $3 \mathrm{f}$ and $2 \mathrm{~b}$ for the structure). At this stage, $g(r)$ is very similar to that obtained for the $2 \mathrm{D}$ silica glass ${ }^{7,8}$. We also calculate the bond angle distribution $(\alpha(\theta))$, presented in Fig. $3 \mathrm{~d}$. Both the spread in the distribution around the ideal hexagon angle $\left(\theta=120^{\circ}\right)$ and the appearance of a second peak close to $108^{\circ}$ are already clear for the structure of Fig. 3 e. These features are further enhanced for the higher disorder case (Fig. $3 \mathrm{f}$ ). The peak at $108^{\circ}$ corresponds to pentagonal carbon rings, which are the second most common topological building blocks of networks built from three-coordinated structural units ${ }^{24}$. Although heptagonal rings are nearly as likely as pentagons, the corresponding peak, which should appear at about $129^{\circ}$, remains hidden within the data. This is caused by local curvature, which allows the larger rings to maintain bonding angles close to $120^{\circ}$.

To obtain a better understanding on changes in the topological order during the transformation, we further calculated the shortest path ring statistics. In Fig. $4 \mathrm{a}-\mathrm{d}$, we plot the results for four different structures: the very first recorded TEM image, at the transition point between the crystalline and disordered phases (close to the structure of Fig. 3 e), for the structure presented in Figs. $2 \mathrm{~b}$ and $3 \mathrm{f}$, and at the completely disordered phase at the end of the experiment (Fig. $2 \mathrm{c}$ ), respectively. A gradually increasing spread is evident during the continuing experiment. Surprisingly, hexagons clearly dominate the statistics even at the highest degree of disorder (Fig. $4 \mathrm{~d}$ ), similar to the random network structure proposed by Shackelford and Brown $^{24}$ and in contrast to the $2 \mathrm{D}$ silica ${ }^{7,8}$. This discrepancy is likely due to the fact that the statistics for 2D silica are based on selected vitreous areas, disregarding crystallites, whereas our analysis was carried out for the complete structure. To facilitate comparison with 


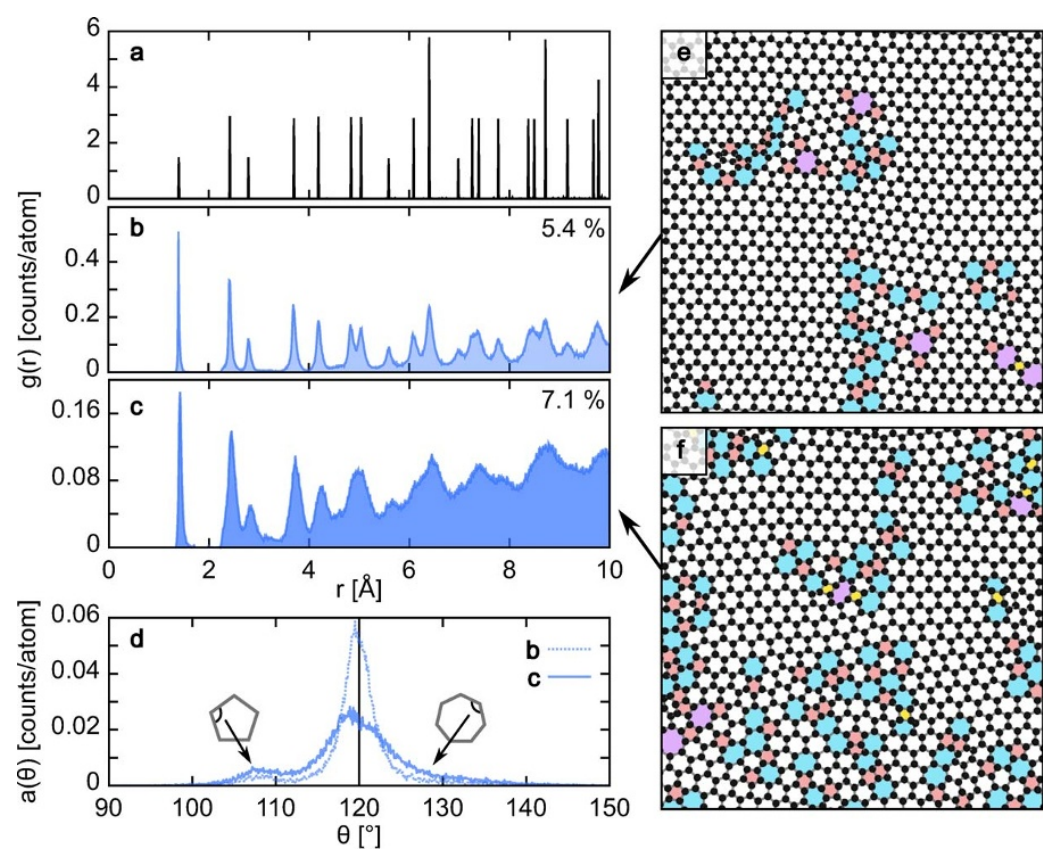

Figure 3 Analysis of the short-range disorder. (a-c) Distribution of the inter-atomic distances (radial distribution function, $g(r)$ ) for pristine graphene, a structure at an intermediate density deficit of ca. 5.4\%, and at a higher density deficit of ca. 7.1\%, respectively. (d) The angular distribution function $(\alpha(\theta))$ for the same structures. Example areas of the structures for the intermediate and high dose cases are shown in panel (e) and (f) with tetragons colored yellow, pentagons red, heptagons blue and octagons violet.

these earlier studies, we have plotted our data in a log normal probability plot (Fig. 4 e). With increasing disorder, our results approach literature values ${ }^{7,8,24}$, with the above-mentioned caveat. As already hinted by the histograms in $4 \mathrm{a}-\mathrm{d}$, our results show that the main change during the experiment is the decreasing ratio of hexagons to other polygons, whereas the ratios between other polygon pairs remain nearly constant. For example, the ratio of pentagons to heptagons is about $1.14 \pm 0.02$ throughout the experiment (this ratio is about 1.3 for the theoretical model of Ref. 24 and between 1.3 and 1.6 for the vitreous $2 \mathrm{D}$ silica $\left.{ }^{7,8}\right)$. This allows for controlled prediction of structural changes made on graphene under an electron beam ${ }^{25}$.

As a final step of the topological analysis, we calculate the ratio of hexagons to all polygons in the structure (i.e., crystallinity, $C^{26}$ ) from ring statistics. For pristine graphene $C=1$, whereas for the vitreous silica structures $C \approx 0.4^{7,8}$. Our dataset, plotted in Fig. $4 \mathrm{f}$, allows for the first time to see the transition between these two extremities. A similar discontinuity, as was already seen in the analysis of the transformation in reciprocal space (Fig. 1 e), appears also within this data: as long as the disorder is limited to defects within the original lattice, crystallinity decreases slowly and linearly. However, as the defects merge into the vitreous network, crystallinity decreases more rapidly, and tends towards a constant ratio of $1 / 1$ between hexagons and nonhexagons (corresponding to $C \approx 0.51$ ). This result supports our earlier hypothesis that the discontinuity seen in Fig. 1 e marks the actual transition point between crystalline graphene and the glassy phase. It also shows that it is possible to create glass structures with different crystallinity values ranging from 0.5 to 0.9 at will. The crystallinity $C$ fits very well to an exponential decay in Fig. 3e, and the final data points are almost at the constant offset $C=0.51$ of this fit. This indicates that we have indeed reached the maximum amount of disorder at the end of the experiment, an equilibrium, where continued randomization of the structure no longer changes its statistical parameters.

Finally, we look at the density fluctuations in the material to establish the amount of order at the longest range. In order to do this, we measure density variation in the final structure (Fig. 2 c). We divide the structure into square sampling areas of different widths $(w=0.2$
.. $2.0 \mathrm{~nm}$ ), and calculate the standard deviation in the calculated densities as a function of $w$ (see Fig. $4 \mathrm{~g}-\mathrm{h}$ ). As expected, the density fluctuations become smaller when the integration size increases, that is, the structure appears smoother on the larger scale. However, as can be seen from the fit in Fig. 4 h, our data follows $1 / w$-behaviour, which is precisely what would be expected for completely random atomic coordinates (or white noise). This indicates the absence of long-range density fluctuations, or presence of hyperuniformity ${ }^{27}$.

In summary, we have reported a solid-state transition from a crystalline mono-layer graphene into a $2 \mathrm{D}$ carbon glass, imposed in a controlled manner using electron irradiation. For the first time, our results shed light into the transitional states between crystalline and amorphous materials, and allow for a controlled creation of 2D carbon glass structures with a specific amount of disorder. The atomic structure of the material is obtained in situ from high-quality TEM images. The transition starts from separated point defects, which merge into a vitreous network separating small crystallites, resembling the crystallite theory of the atomic structure of a glass. At the final stage, according to both real space and reciprocal space analysis, the structure is indistinguishable from a $2 \mathrm{D}$ random network. Our study shows that the two competing theories of the structure of glass, the nano-crystalline theory and the random network model, provide complementary descriptions on the same material, each applying to a specific range of disorder, at least in two dimensions.

\section{Methods}

Our graphene samples were prepared by mechanical exfoliation with subsequent transfer to a TEM grid. An image side aberration corrected FEI Titan 80-300 was aligned for high resolution imaging at $100 \mathrm{kV}$. Images were recorded at a Scherzer defocus and the spherical aberration was set to ca. $20 \mu \mathrm{m}$. Under these conditions, the dark contrast can be directly interpreted as the atomic structure. The TEM image sequence of the amorphization was shown before (supplementary information of Refs. 12,13), but no analysis of the amorphous areas as shown here was done previously.

Energy minimization and structural optimization was carried out with the conjugate gradient method as implemented in the LAMMPS simulation package ${ }^{28,29}$. For the radial and angular distribution analysis, the obtained structures (which had about 13,000 atoms with sizes ca. $30 \times 30 \mathrm{~nm}^{2}$ ) were embedded in a larger ideal lattice (with more than twice the number of atoms) to ensure realistic relaxation also at the edges 

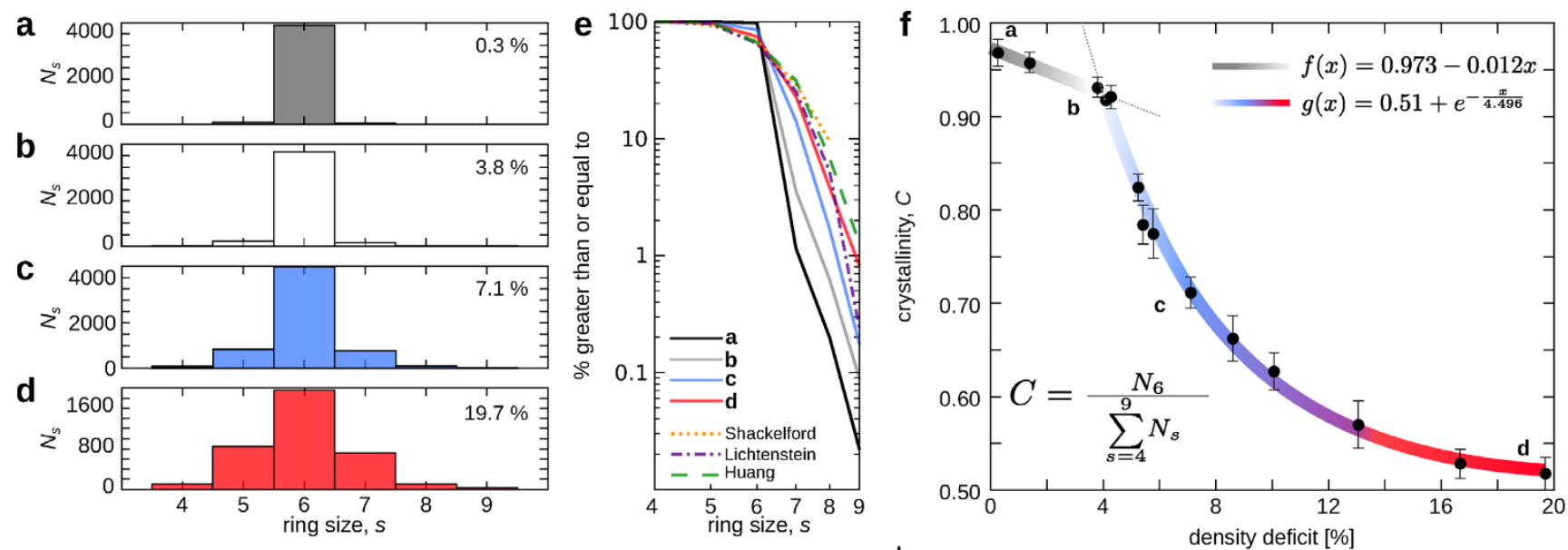

g

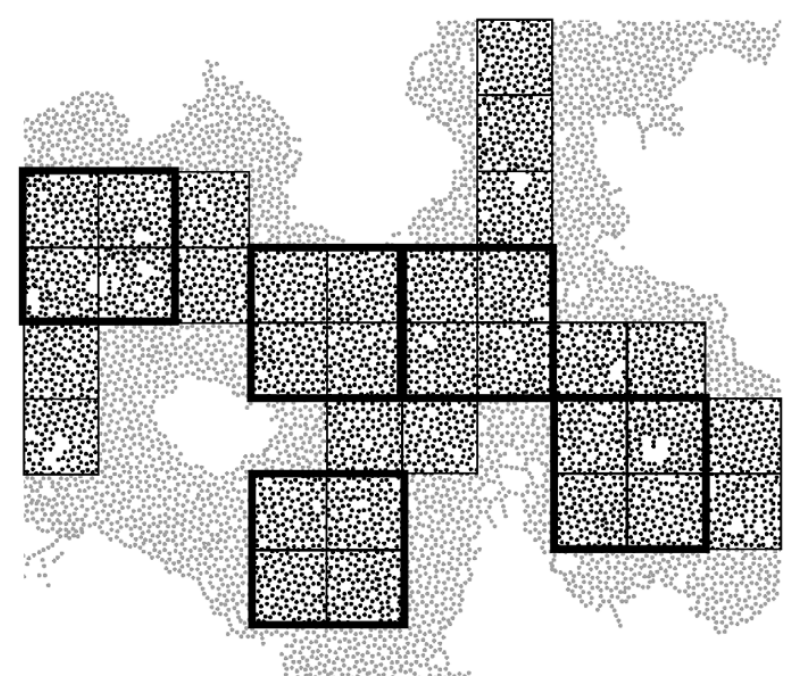

$\mathrm{h}$

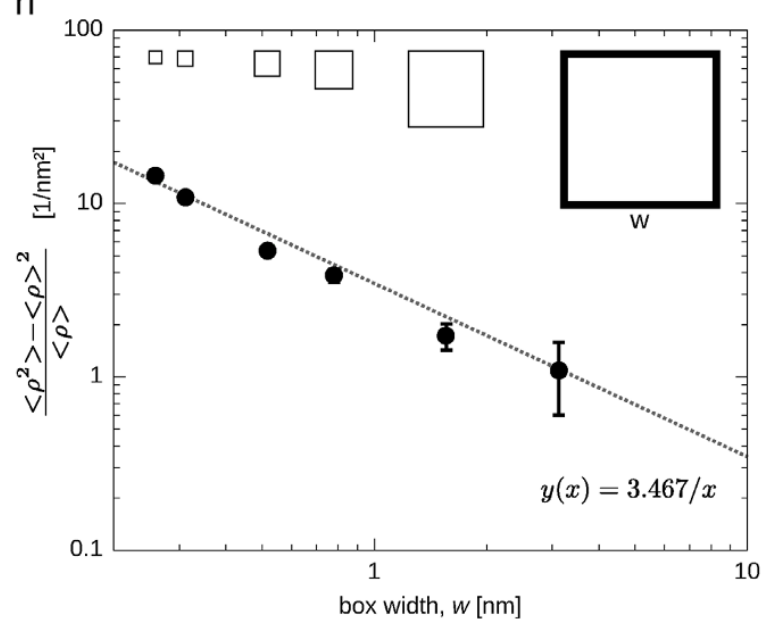

Figure $4 \mid$ Topological order and long-range density fluctuations. (a-d) The ring size statistics for the very first recorded TEM image (density deficit of $0.3 \%)$, at the transition point between crystalline and disordered states (3.8\%), at a density deficit of $7.1 \%$ and at the end of the experiment (19.7\%), respectively. (e) Log-normal probability distribution of the ring statistics for the same structures and three literature results (from Refs. 7,8,24). (e) Crystallinity, as calculated from ring statistics as a function of the density deficit. The color bar indicates the transition from the ordered (gray) via nanocrystallite (blue) to a random network (red). (g) Structure of the disordered carbon glass corresponding to a density deficit of ca. 19.7\%, with selected sampling areas (avoiding larger holes and edges) for the analysis of density variations. The largest and second largest sampling sizes are shown; smaller ones are obtained by dividing these areas. (h) Density fluctuations at different scales. The plot shows the atom number density $(\rho)$ standard deviation among the boxes versus the box width $(w)$. The $1 / w$ dependence, as shown by the dotted line, indicates the behaviour that would be expected for a completely random structure or white noise. The inset shows the sampling areas corresponding to the data points at the same scale as panel (g).

of the field of view of the experimental images. This ideal lattice was excluded from the analysis after structural optimization. All structures (optimized without the surrounding lattice) used for the shortest path ring statistics are provided as supplementary material. For this analysis, we excluded all edges to minimize the errors in the interpretation caused by adsorbates and holes in the structure. The carbon-carbon interactions were modeled with the AIREBO potential ${ }^{30}$, as implemented in LAMMPS. Any inaccuracies stemming from the use of a semi-empirical interaction model are expected to be limited to the absolute values of the inter-atomic distances. Therefore, they have no influence on any of the conclusions made in the study.

1. Anderson, P. Through the Glass Lightly. Science 267, 1615-1616 (1995).

2. Wright, A. C. Neutron scattering from vitreous silica. V. The structure of vitreous silica: What have we learned from 60 years of diffraction studies? J. Non Cryst. Sol. 3093 (1994).

3. Wright, A. C. \& Thorpe, M. F. Eighty years of random networks. Phys. Stat. Sol. (B) 250, 931-936 (2013).

4. Zachariasen, W. The Atomic Arrangement in Glass. Journ. Am. Chem. Soc 54, 3841-3851 (1932).

5. Kumar, A., Wilson, M. \& Thorpe, M. F. Amorphous graphene: a realization of Zachariasen's glass. J. Phys.: Condens. Matter 24, 485003 (2012).

6. Sheng, H. W., Luo, W. K., Alamgir, F. M., Bai, J. M. \& Ma, E. Atomic packing and short-to-medium-range order in metallic glasses. Nature 439, 419-25 (2006).

7. Lichtenstein, L. et al. The atomic structure of a metal-supported vitreous thin silica film. Angew. Chem. Int. ed. in English 51, 404-7 (2012).
8. Huang, P. Y. et al. Direct Imaging of a Two-Dimensional Silica Glass on Graphene. Nano Lett. 12, 1081-1086 (2012).

9. Gibson, J. M., Treacy, M. M. J., Sun, T. \& Zaluzec, N. J. Substantial Crystalline Topology in Amorphous Silicon. Phys. Rev. Lett. 105, 125504 (2010).

10. Stillinger, F. H. A topographic view of supercooled liquids and glass formation. Science 267, 1935-9 (1995).

11. Turnbull, D. \& Cohen, M. H. Concerning Reconstructive Transformation and Formation of Glass. J. of Chem. Phys. 29, 1049 (1958).

12. Kotakoski, J., Krasheninnikov, A., Kaiser, U. \& Meyer, J. From Point Defects in Graphene to Two-Dimensional Amorphous Carbon. Phys. Rev. Lett. 106, 105505 (2011).

13. Meyer, J. et al. Accurate Measurement of Electron Beam Induced Displacement Cross Sections for Single-Layer Graphene. Phys. Rev. Lett. 108, 196102 (2012).

14. Kotakoski, J. et al. Stone-Wales-type transformations in carbon nanostructures driven by electron irradiation. Phys. Rev. B 83, 245420 (2011).

15. Terrones, H. et al. New Metallic Allotropes of Planar and Tubular Carbon. Phys. Rev. Lett. 84, 1716-1719 (2000).

16. Crespi, V., Benedict, L., Cohen, M. \& Louie, S. Prediction of a pure-carbon planar covalent metal. Phys. Rev. B 53, R13303-R13305 (1996).

17. Patterson, A. The Scherrer formula for X-ray particle size determination. Phys. rev. 56, 978-982 (1939).

18. Lehtinen, O., Kurasch, S., Krasheninnikov, A. V. \& Kaiser, U. Atomic scale study of the life cycle of a dislocation in graphene from birth to annihilation. Nat. comm. 4, 2098 (2013).

19. Westenfelder, B. et al. Transformations of Carbon Adsorbates on Graphene Substrates under Extreme Heat. Nano lett. 11, 5123-5127 (2011). 
20. Turchanin, A. et al. Conversion of self-assembled monolayers into nanocrystalline graphene: structure and electric transport. ACS nano 5, 3896-904 (2011).

21. Huang, P. Y. et al. Grains and grain boundaries in single-layer graphene atomic patchwork quilts. Nature 469, 389-92 (2011).

22. Kim, K. et al. Grain boundary mapping in polycrystalline graphene. ACS nano 5 , 2142-6 (2011).

23. Kurasch, S. et al. Atom-by-atom observation of grain boundary migration in graphene. Nano lett. 12, 3168-73 (2012).

24. Shackelford, J. \& Brown, B. The Lognormal Distribution in the Random Network Structure. J. Non-Cryst. Sol. 44, 379-382 (1981).

25. Robertson, A. W. et al. Spatial control of defect creation in graphene at the nanoscale. Nat. comm. 3, 1144 (2012).

26. Lichtenstein, L., Heyde, M. \& Freund, H.-J. Crystalline-Vitreous Interface in Two Dimensional Silica. Phys. Rev. Lett. 109, 106101 (2012).

27. Torquato, S. \& Stillinger, F. Local density fluctuations, hyperuniformity, and order metrics. Phys. Rev. E 68, 041113 (2003).

28. Plimpton, S. Fast Parallel Algorithms for Short-Range Molecular Dynamics. J. Comp. Phys. 1-19 (1995).

29. Grippo, L. \& Lucidi, S. A globally convergent version of the Polak-Ribiere conjugate gradient method. Math. Prog. 78, 375-391 (1997).

30. Stuart, S. J., Tutein, A. B. \& Harrison, J. A. A reactive potential for hydrocarbons with intermolecular interactions. J. Chem. Phys. 112, 6472 (2000).

\section{Acknowledgments}

We acknowledge funding from the Austrian Science Fund (FWF: M1481-N20 and I1283-N20), the German Ministry of Science (DFG), Research and the Arts (MWK) of the State of Baden-Wuertternberg within the SALVE (Sub-Angstrom Low-Voltage Electron microscopy) project, and University of Helsinki Funds, as well as computational time from the Vienna Scientific Cluster.

\section{Author contributions}

F.R.E. and J.K. contributed equally to this study. F.R.E. and J.K. did the analysis, developed the method for extracting atomic coordinates with J.C.M., and carried out the simulations. J.C.M. carried out experiments. F.R.E., J.K. and J.C.M. wrote the paper. U.K. and J.C.M. supervised the work. All authors commented on the manuscript.

\section{Additional information}

Supplementary information accompanies this paper at http://www.nature.com/ scientificreports

Competing financial interests: The author(s) declare no competing financial interests.

How to cite this article: Eder, F.R., Kotakoski, J., Kaiser, U. \& Meyer, J.C. A journey from order to disorder - Atom by atom transformation from graphene to a $2 \mathrm{D}$ carbon glass. Sci. Rep. 4, 4060; DOI:10.1038/srep04060 (2014)

This work is licensed under a Creative Commons AttributionNonCommercial-ShareAlike 3.0 Unported license. To view a copy of this license, visit http://creativecommons.org/licenses/by-nc-sa/3.0 